\title{
Incremental voting based spectrum sensing model for cognitive radio networks
}

\author{
Sufyan Muhammad Khan', Waqas Muhammad Khan², Fazeel Uddin Faraz ${ }^{3}$, Sajjad Muhammad Khan \\ ${ }^{1}$ Department of Computing and Technology, Iqra University, Islamabad 44000, Pakistan \\ ${ }^{2}$ Department of Physics, International Islamic University, Islamabad 44000, Pakistan \\ ${ }^{3}$ Department of Computer Science, International Islamic University, Islamabad 44000, Pakistan \\ ${ }^{4}$ National Center of Excellence in Geology, University of Peshawar, Peshawar 25000, Pakistan
}

Corresponding Author Email: waqaskhanrwp@yahoo.com

https://doi.org/10.18280/rces.050201

Received: 28 April 2018

Accepted: 30 June 2018

\section{Keywords:}

opportunistic spectrum sensing, poling scheme for cognitive radio, voting based spectrum sensing, Cognitive Radio Networks (CRN)

\begin{abstract}
Cognitive Radio Networks offers solution of spectrum insufficiency, In CRNs licensed spectrum channels are used by licensed user (Primary users) and unlicensed users (Secondary users) such that secondary user (SU) does not harm and interfere activities of primary user (PU). Spectrum Decision System is required for intelligent spectrum sensing, access and distribution between PU and SU. Cooperative spectrum sensing (CSS) can reduce the overhead on signal processing techniques and enable the SUs for reliable detection of PUs activity. Spectrum availability in CSS approach node makes a binary decision based on its local observation and then forwards its decision to the fusion center (FC). At the FC, all these decisions are merged together according to some fusion rule.

In this paper, a frameork for cooperative SUs is presented, which have to decide about the presence or absence of the PU in target spectrum. The performance of the framework is then evaluated and it is observed that it outperforms different commonly used routing scheme. It ensures to sufficiently increase the probability of correct detection and decrease in the probability of false alarm.
\end{abstract}

\section{INTRODUCTION}

Federal Communications Commission (FCC) has concluded in its recent study that fixed spectrum assignment policy becomes a bottleneck for more efficient spectrum utilization, under which a great portion of the licensed spectrum is under-utilized. Such inefficient usage of the limited spectrum demands the development of dynamic spectrum access (DSA) techniques.

Cognitive radio (CR) is the promising technology that enables next generation communication networks, also known as DSA networks, to utilize the spectrum more efficiently in an opportunistic fashion without interfering with the PUs. The CR facilitates the usage of temporally and spatially unused spectrum, which is referred to as spectrum hole or white space. In order words these spectrum holes can be present not only at certain geographical position but also for particular time intervals [1-5].

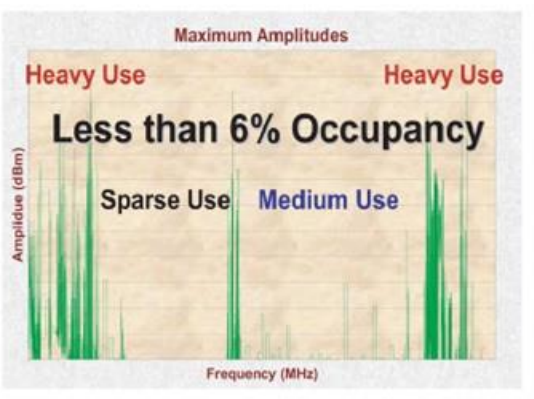

Figure 1. Spectrum usage [1]

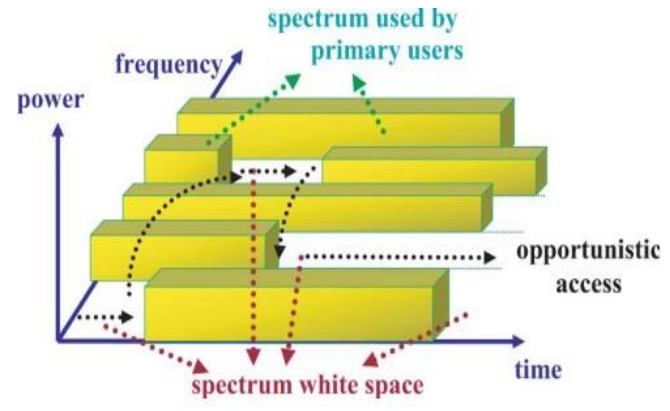

Figure 2. Temporal spectrum hole concept [1]

This spatial or temporal unused licensed spectrum is then available to be utilized by SUs. The temporal and spatial spectrum holes.

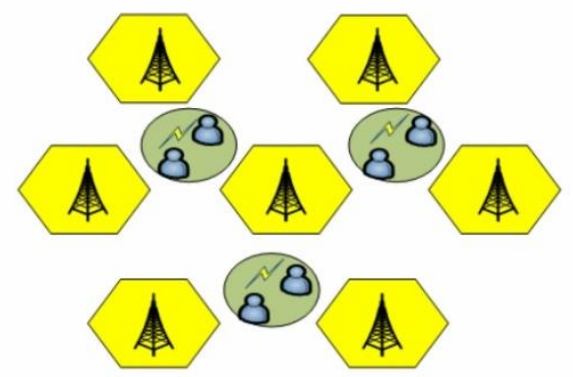

Figure 3. Spatial spectrum hole concept [6]

CRs sense and adapt itself to the target radio frequency 
environment in order to utilize spectrum holes. Reliable spectrum sensing is an important feature of CRs which enables them for efficient and interference free spectrum access during idle state of PUs [7]. Unfortunately reliable spectrum sensing package comes up with great challenges, one of them is hidden terminal problem.

Figure 4 depicts the hidden terminal problem where CRs are unable to detect the presence of PUs due to shadowed environment in drastic multipath fading (a.k.a fast fading). This problem results in harmful or severe interference to the PUs. Recent research suggests that CSS or DSS can easily mitigate the hidden terminal issue. Cooperative sensing improves the performance of spectrum sensing through multiple cooperative CRs [6-10].

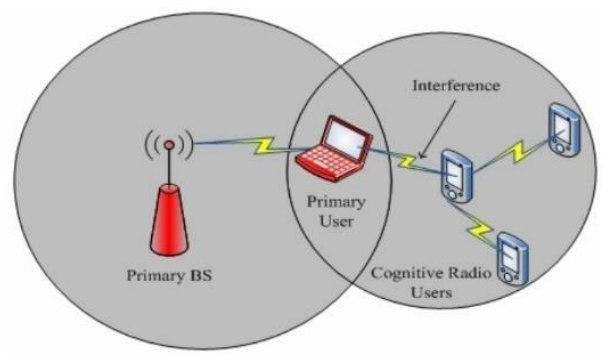

Figure 4. Hidden terminal problem in CRN [6]

Optimized fusion rule framework for cooperative SUs has been introduced in this paper, to help detect PUs reliably. The performance of the optimized fusion rule framework is then compared with existing fusion rules. Simulation results shows that our fusion rule outperforms different commonly used fusion rules e.g. AND or OR rule. It also ensures to sufficiently increase the probability of correct detection by inducing decrease in the probability of false opportunity detection.

The rest of the paper is organized as follow, second section provide related work, third section provide proposed method, fourth section provide proposed algorithm, fifth section provide results, sixth section provide conclusion and last section provide future work.

\section{RELATED WORK}

To identify the PU activity in CRN is the most important task for SU. The detection of PU is termed as spectrum sensing, in CSS polling is one method used for detecting PU in spectrum, in polling all SU participate in deciding whether PU is present and transmitting, this decision is based on PU activity sensed by each SU present in CRN [11]. It is highly likely that there might be some compromised nodes termed as malicious users which sends false sensing data, as a result FC which is a central location for collecting spectrum sensing information receive wrong data and can take wrong spectrum sensing decision hence the performance of cooperative sensing schemes can be severely affected.

Due to the fact that every SU participate in polling process, the sensing time of decision making process is relatively larger than the transmission time. Furthermore, the SU consumes time in handshake and establishing the path $[12,16,20]$. To do this all procedure, most of the time consumed by the SU in all these steps is much greater than their actual transmission time.

Furthermore the detection of the SUs or nodes is not properly done or is affected by some other factors. The malfunctioning in $\mathrm{CRN}$ is occurred due to different factors e.g. false alarms etc.

\section{PROPOSED METHOD}

To optimize time consume in polling process for spectrum sensing, we are going to introduce Incremental Polling Scheme for CSS in CRN. Our first and foremost goal to sense the spectrum in less time and transmit the data for maximum time, in short our aim is to maximize the transmission time. In proposed solution, basic theme of our proposed strategy is instead of waiting sensing result from $100 \%$ nodes, if we found a strong pattern in early stages of results collection than a decision can be taken without waiting for the rest of nodes, this strategy will not only save polling time for the rest of the results from sensing nodes in network but also broaden the transmission time for nodes in the network.

Our proposed incremental polling scheme based on the idea of decision region initially this decision region wait for reply from $30 \%$ of nodes in network, a threshold level (which is $80 \%$ ) is also defined, if decision region found $80 \%$ of nodes response in same fashion (either yes or no) it will not wait for rest of the responses from other $\mathrm{SU}$, takes a decision and send decision regarding spectrum availability to waiting SU, if 30\% nodes in decision region does not meet threshold level than $10 \%$ more nodes included in this region, this ten percent of node increment continues until our required threshold level achieved or all nodes result received proposed scheme is illustrated in the form of figure 6 .

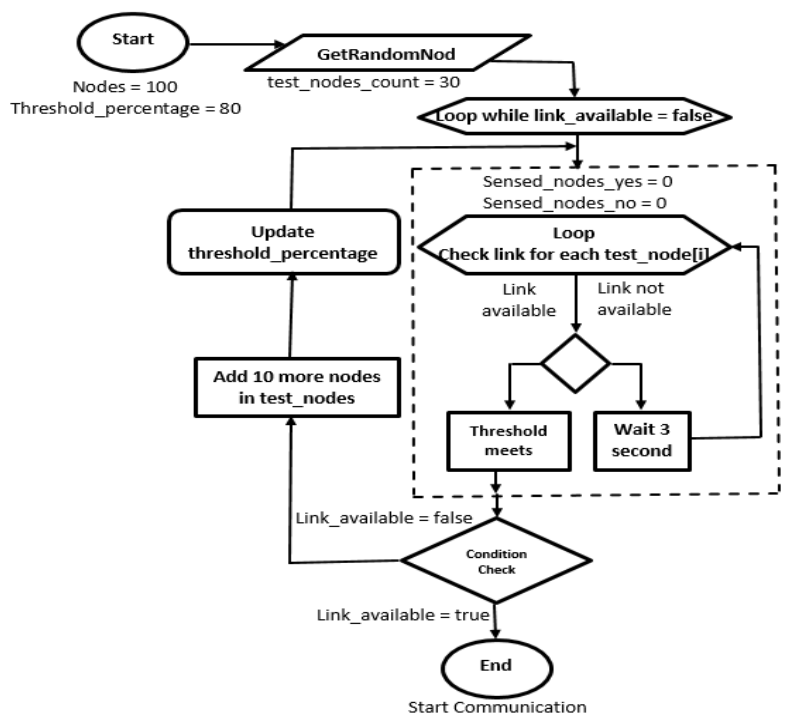

Figure 5. Incremental voting based spectrum sensing model for CRN

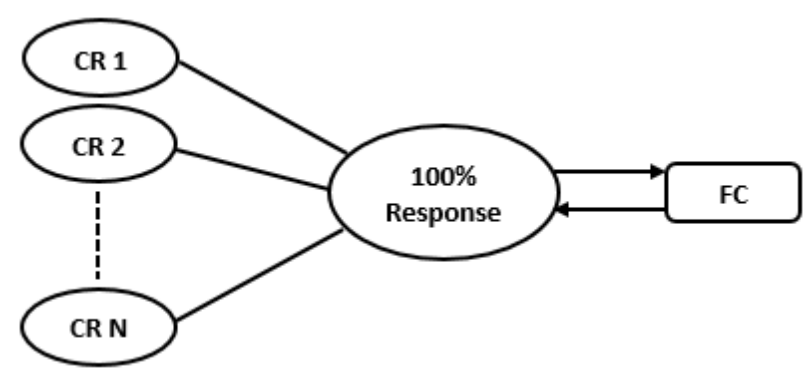

Figure 6. Basic incremental polling scheme 


\section{RESULTS}

\subsection{System model}

4.1.1 Cognitive radio sensor network parameters:

Following parameters for cognitive radio sensor network are used

$$
\begin{aligned}
& \text { - Cooperative sensing technique: } \\
& \text { Centralized } \\
& \text { - Spectrum sensing technique: Energy } \\
& \text { detection } \\
& \text { - Nodes: } 100 \text { sensing nodes (SUs) }
\end{aligned}
$$

4.1.2 Environment

In simulation, the following environment are used

- Channel type: Multipath with Rayleigh fading

(Bluetooth)

PU signal type: $1 \mathrm{MHz}$ BW GMSK

- $\quad$ Maximum data rate: $\mathrm{R}=1 \mathrm{Mbps}$

- $\quad$ Sensing time: Ts $=1 \mathrm{~ms}$.

- $\quad$ Reporting time of each SU: $\mathrm{Tr}=1 \mathrm{~ms}$

- $\quad$ Sensing period: $\mathrm{Tsp}=1 \mathrm{~s}$.

\subsubsection{Algorithm}

- $\quad$ There are 2 PUs

- 100 nodes (sensing nodes/SUs) for detecting the spectrum

- 1 node/FC is used for decision making

- $\quad$ CSS scheme - sequential.

- Decision making type - hard decision for $\mathrm{K}$ out of $\mathrm{M}$ rule. Threshold level for sensing the link $\mathrm{b} / \mathrm{w}$ PUs either IDLE or OCCUPIED by sensing nodes/SUs:

1. $80 \%$ threshold level for 1 to 50 sensing nodes (SUs)

2. $75 \%$ threshold level for 51 to 70 sensing nodes (SUs)

3. $70 \%$ threshold level for 71 to 100 sensing nodes (SUs)

\subsubsection{Scenarios}

There are basically two scenarios on which the given algorithm will be implementing

1. When the link is idle/free (both the PUs do not communicate/transmitting data)

2. When the link is occupied (both the PUs

\begin{tabular}{|c|c|c|c|}
\hline & $\begin{array}{c}\text { Maximum Data } \\
\text { Rate }\end{array}$ & \multicolumn{2}{|c|}{$1 \mathrm{Mbps}$} \\
\hline & Sensing Time & \multicolumn{2}{|c|}{$1 \mathrm{~ms}$} \\
\hline & Reporting Time & \multicolumn{2}{|c|}{$1 \mathrm{~ms}$} \\
\hline & Sensing Period & \multicolumn{2}{|c|}{$1 \mathrm{~s}$} \\
\hline \multirow[t]{8}{*}{ Algorithm } & \multicolumn{3}{|c|}{2 Pus } \\
\hline & \multicolumn{3}{|c|}{100 Sensing Nodes } \\
\hline & \multicolumn{3}{|c|}{1 FC (Decision Making Node) } \\
\hline & $\begin{array}{c}\text { Co-operative } \\
\text { Spectrum Sensing } \\
\text { Scheme }\end{array}$ & \multicolumn{2}{|c|}{ Sequential } \\
\hline & $\begin{array}{c}\text { Decision Making } \\
\text { Type }\end{array}$ & \multicolumn{2}{|c|}{ K out of M Rule } \\
\hline & \multirow[t]{3}{*}{ Threshold Level } & $\begin{array}{c}1 \text { to } \\
50\end{array}$ & $80 \%$ \\
\hline & & $\begin{array}{c}51 \text { to } \\
70\end{array}$ & $75 \%$ \\
\hline & & $\begin{array}{c}71 \text { to } \\
100\end{array}$ & $70 \%$ \\
\hline \multirow[t]{2}{*}{ Scenario } & \multicolumn{3}{|c|}{ Link is Free } \\
\hline & \multicolumn{3}{|c|}{ Link is Occupied } \\
\hline
\end{tabular}
communicate/transmitting data).

Table1. Parameter used in cognitive radio network environment

\begin{tabular}{|c|c|c|}
\hline $\begin{array}{c}\text { Cognitive Radio } \\
\text { Sensor Network } \\
\text { Parameters }\end{array}$ & $\begin{array}{c}\text { Co-operative } \\
\text { Sensing } \\
\text { Technique }\end{array}$ & Centralized \\
\cline { 2 - 3 } & $\begin{array}{c}\text { Spectrum } \\
\text { Sensing } \\
\text { Technique }\end{array}$ & Energy Detection \\
\cline { 2 - 3 } & Nodes & $\begin{array}{c}100 \text { sensing nodes } \\
\text { (SU) }\end{array}$ \\
\hline \multirow{2}{*}{ Environment } & Channel Type & $\begin{array}{c}\text { Multipath Rayleigh } \\
\text { Fading }\end{array}$ \\
\cline { 2 - 3 } & PU Signal Type & $\begin{array}{c}1 \mathrm{MHz} \text { BW } \\
\text { (Bluetooth) }\end{array}$ \\
\hline
\end{tabular}

\subsection{Mathematical model}

Let's describe model of given system. Two PU establish operational area in form of circle of radius which is equal to distance between PU. SU are uniformly distributed among this area for all studying cases of numbers of active 30, 50, 70 and 100. Since CSS is used we will perform studying for Rayleigh fading channel, where it provides gain. Distance between PU not so big, thus amplifiers of all devices can compensate general signal pass loss. But since fading is present amplitude of signals at all SU are depended on channel impulse response $\boldsymbol{h}=\boldsymbol{\alpha} \boldsymbol{e}^{-j \boldsymbol{\varphi}}$, where $\boldsymbol{\alpha}$-cumulativeamplitude gain of channel, $\boldsymbol{\varphi}-$ cumulative phase shift of channel. Since $h$ to each SU are dispersed by Rayleigh probability distribution, then signals at all SU also has been dispersed in the same way. In paper [13] signals at SU input at fading channel for both of scenarios of link idle with probability $\mathrm{HO}$ and link occupied with probability $\mathrm{H} 1$ are described as

$E \approx\left\{\begin{array}{cc}\chi_{2(T W+1)}^{2}, & H_{0} \\ e_{2\left(\gamma^{2}+1\right)}+\chi_{2 T W}^{2}(\lambda), & H_{1},\end{array}\right.$

where $\chi_{2 T W}^{2}(\lambda)$ - random value of chi-square distribution with degrease of freedom $2 \mathbf{T W}$ and non-centrality parameter

$\lambda$ (according to [14] $\lambda=\mathbf{2} \gamma), e_{2\left(\gamma^{2}+1\right)}$ - random value of exponential distribution with parameter (mean value) $\mathbf{2}\left(\gamma^{2}+\mathbf{1}\right)$, $\gamma$-desirable signal to noise rate (SNR), TW - multiplication of sampling/sensing time per bandwidth or number of samples has been taken for decision making.

Chi-square random value describes noise signal and exponential random value describes PU signal at SU input. Thus real SNR at SU input will be calculated as $\bar{\gamma}=e_{2\left(\gamma^{2}+1\right)} / \chi_{2 T W}^{2}(\lambda), H_{1}$ for occupied link and $\bar{\gamma}=1, H_{0}$ for idle link.

\subsubsection{Mathematical model for $\mathrm{P}_{\mathrm{d}}$ and $\mathrm{P}_{\mathrm{f}}$}

Parameters that describes occupied link are probability of misdetection $\mathbf{P}_{\mathbf{m}}$ and probability of detection $\mathbf{P}_{\mathbf{d}}$. Parameter that describe idle/free link is probability of false alarm $P_{f}$. 
Thereby we will use $P_{d}$ for describing occupied link scenario and $P_{f}$ for describing idle/free link scenario.

According to [15] $P_{d}$ and $P_{f}$ for fading channel can be derived as:

$$
P_{d}=e^{-\frac{\psi}{2}} \sum_{m=0}^{T W-2} \frac{1}{m !}\left(\frac{\psi}{2}\right)^{m}+\left(\frac{1+\bar{\gamma}}{\bar{\gamma}}\right)^{T W-1}\left[e^{-\frac{\psi}{2(1+\bar{\gamma})}}-e^{-\frac{\psi}{2}} \sum_{m=0}^{T W-2} \frac{1}{m !}\left(\frac{\psi \cdot \bar{\gamma}}{2(1+\bar{\gamma})}\right)^{m}\right]
$$

$$
P_{f}=\frac{\Gamma(T W, \psi / 2)}{\Gamma(T W, 0)}
$$

where

$$
\begin{aligned}
& \bar{\gamma} \text { - actual signal to noise ratio at receiver input, } \\
& \Gamma(\cdot, \cdot) \text { - incomplete gamma function, } \\
& \psi-\text { RX energy threshold. }
\end{aligned}
$$

\subsubsection{Mathematical model for cooperative spectrum sensing}

Given CSS algorism is described by $\mathrm{K}$ out of $\mathrm{M}$ rule, according to [16] and use hard decision making to reduce link throughput usage while SU reporting to FC node. FC is located at one point with one of PU.

According [17-18] probabilities of success decision and false alarm for CSS at hard decision for $\mathrm{K}$ out of $\mathrm{M}$ rule are:

$$
P_{D}=\sum_{k=0}^{M-K} \frac{M !}{(K+k) !(M-K-k) !}\left(1-P_{d, k}\right)^{M-K-k}\left(P_{d, k}\right)^{K+k},
$$

$$
P_{F}=\sum_{k=0}^{M-K} \frac{M !}{(K+k) !(M-K-k) !}\left(1-P_{f, k}\right)^{M-K-k}\left(P_{f, k}\right)^{K+k}
$$

where $\mathrm{M}$ - total number of SU, K - threshold number of SU that performs the decision

\subsection{Efficiency of cooperative spectrum sensing}

Efficiency of CSS can be described as ratio of obtained result at SU or FC to spent resource. Resource that is spent is energy in Joules $(J)$. Result for SU is useful data rate/throughput, for $\mathrm{FC}$ is useful data rate/throughput too or probability of right decision. Thus efficiency of SU or FC is measured as Bps/J and for FC is effectively used energy in $\mathrm{J}$. In CSS argument of efficiency equation is number of used SU for decision making. Thereby in this paragraph we will study dependence Efficiency vs Number of used SU.

First of all we review time spending procedure since TDMA usage is assumed for SU polling/reporting. Generally time frame format is used for CR as the best separation method for PU and SU transmission, as in fig.2. Each time frame consists of sensing time $T_{s}$, reporting time $T_{r}$ and transmission time $T_{t}$, as in fig.8. Total frame time described as:

$$
T_{F T}=T_{s}+T_{r} \cdot N+T_{t},
$$

where $N-$ total number of SU that are polled.

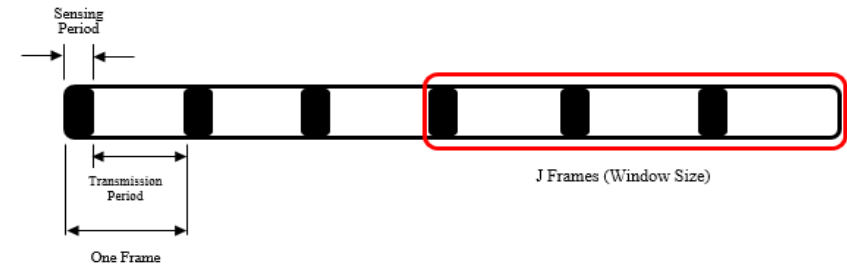

Figure 7. Separation method for PU and SU transmission

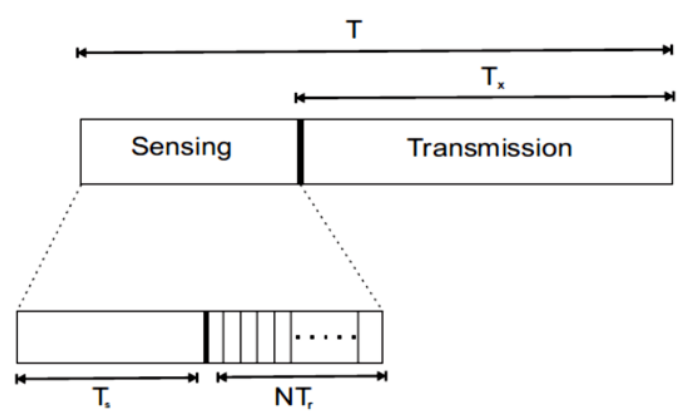

Figure 8. Time hen

We can derive equation for throughput. Since SU can transmit signal at maximum rate $\mathrm{R}$ during time $T_{t}$ when $\mathrm{PU}$ do not transmit signal with probability $P\left(H_{0}\right)$ and $\mathrm{FC}$ do not produce false alarm with probability of $\left(1-P_{F}\right)$, then:

$C=\frac{T_{t}}{T_{F T}} \cdot R \cdot\left(1-P_{F}\right) \cdot P\left(H_{0}\right)$

Before efficiency calculation let's define energies that are consumed by SU and FC.

\subsubsection{Energy consumption}

The energy consumption (EC) of different scenarios are given as follow

- EC of one SU sensing $E_{S S}=T_{s} \cdot w_{s}$, where $w_{s}$ - unit power of SU sensing;

- EC of one SU reporting $E_{S R}=T_{r} \cdot w_{r}$, where $w_{r}$ - unit power of SU reporting;

- EC of FC reporting $E_{F R}=N \cdot T_{r} \cdot w_{r}$, where $w_{r}$ - unit power of $\mathrm{FC}$ reporting managing;

- EC of SU transmitting $E_{S T}=T_{t} \cdot w_{t}$, where $w_{t}$ - unit power of SU data transmission;

Then total consumption energy of SU is $E_{S}=E_{S S}+E_{S R}+E_{S T}$ and for $\mathrm{FC}$ is $E_{F}=E_{F R}$.

At last, we can derive equations for energy efficiency of SU and FC. Efficiency for SU uniquely defined as:

$\varepsilon_{S}(N)=\frac{C}{E_{S}}=\frac{T_{t} / T_{F T} \cdot R \cdot\left(1-P_{F}\right) \cdot P\left(H_{0}\right)}{T_{s} \cdot w_{s}+T_{r} \cdot w_{r}+T_{t} \cdot w_{t}}$

Efficiency for FC in the concept of probability of right decision is:

$\varepsilon_{F}(N)=P_{D}\left(1-P_{F}\right) E_{F}=P_{D}\left(1-P_{F}\right) \cdot N \cdot T_{r} \cdot w_{r}$ 
From another point of view this equation can be expressed as $\varepsilon_{F}(N)=P_{D}\left(1-P_{F}\right)$, so it is not depended on consumed energy, then we derive it by using parameter of achievable throughput for SU as:

$\varepsilon_{F}(N)=\frac{C}{E_{F}}=\frac{T_{t} / T_{F T} \cdot R \cdot\left(1-P_{F}\right) \cdot P\left(H_{0}\right)}{N \cdot T_{r} \cdot w_{r}}$

\subsection{Simulation results}

In this paragraph MATLAB simulation results are presented.

The main characteristics of SU in cognitive radio are $P_{d}$ and $P_{f}$ that describe SU performance at occupied and idle link cases respectively. Usually [19], $P_{d}$ and $P_{f}$ are studied as dependence from RX threshold level. As first step, we need to calculate TW, $\psi, \gamma$ before $P_{d}$ and $P_{f}$ will be calculated from (2) and (3) respectively. The $\psi$ can be obtained as SNR at lower bound of BER $=10^{-2}$ for GMSK that is chosen as PU signal, thus $\psi=6.3(8 \mathrm{~dB}) . \gamma$ can be obtained as SNR at average common value or upper bound of $\mathrm{BER}=10^{-3}, 10^{-6}$ for GMSK, thus $\gamma=10$ or $25(14 \mathrm{~dB})$. Let's take $\gamma=10$ as mean SNR of receiving signals at SU. TW can be obtained in iterative way while $\psi$ is calculated from equation that is inversed to (3): $\psi=2 \Gamma^{-1}(\mathrm{Pf}=0.1, \mathrm{TW})$. At $\mathrm{TW}=3, \psi \approx 6.3$. Then we can calculate probabilities for occupied and idle link scenarios for different numbers of $\mathrm{SU}=30,50,70,100$.

For studying of CSS effectiveness lets show cumulative $P_{d}$ and $P_{f}$ results for different SU number on one figure, as in fig.9(a) and 9(b) respectively.

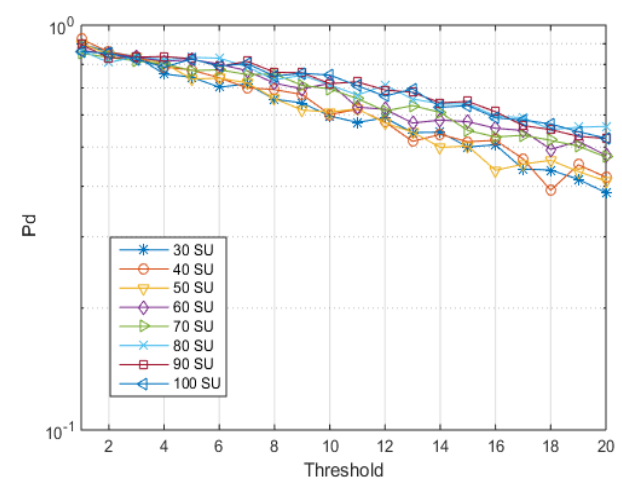

a)

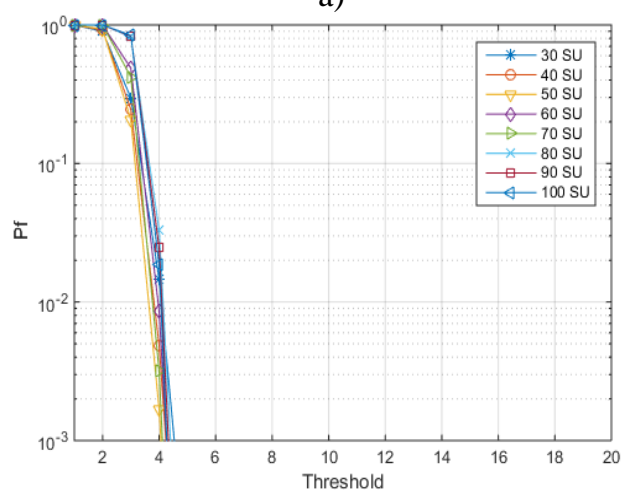

b)

Figure 9. (a, b) Cumulative $P_{d}$ and $P_{f}$ results for different SU number
From fig.4 it can be observed that with increasing of threshold both of $P_{d}$ and $P_{f}$ are decreasing, because probability of high amplitude noise and desired signal at high threshold is lower than low amplitude noise and desired signal at low threshold.

From fig.9, it's hard to understand how number of SU influences on $P_{d}$ and $P_{f}$, then let's calculate average values of $P_{d}$ and $P_{f}$ among all thresholds for each case of SU numbers. Results for this study of $P_{d}$ and $P_{f}$ are shown in fig.10 (a) and 10 (b) respectively.

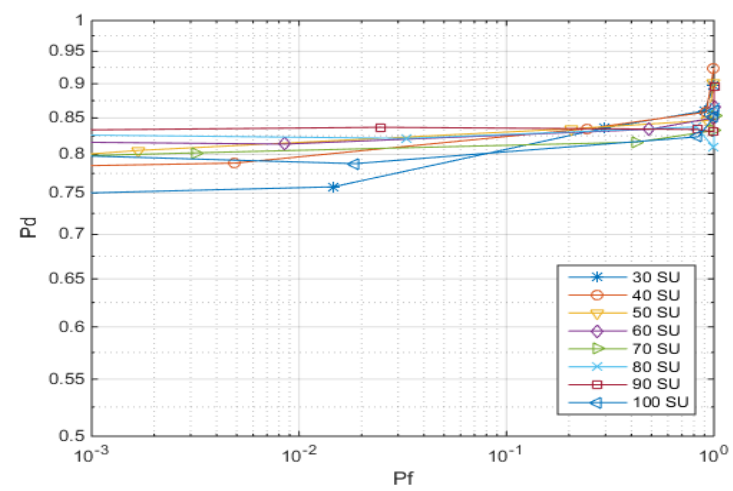

Figure 11. Cumulative results for all ROC

From fig.10 it's can be observed that while number of SU increase performances of SU are decreased and while threshold level of SU numbers is decreased performances of SU are increased.

Another main characteristic of $\mathrm{SU}$ in $\mathrm{CR}$ is receiver operating characteristic (ROC) that describes probability dependencies of successive and false detection. Usually it is performed as rate of $P_{d}$ to $P_{f}$ at the same threshold while signal with some SNR is received. In this simulation receiving of signals with mean SNR $=10$ by $30,40 \ldots 100 \mathrm{SU}$ was performed. Separate ROC for 30, 50, 70 and $100 \mathrm{SU}$ cases at mean SNR $=10$ of received signal. Cumulative results of this simulation are shown in fig. 11.

All mentioned results before was completed as average of 100 studying cases.

From fig. 11 we can conclude that all dependencies from fig. 9 and 10 are saved, also if mean SNR is higher than desired threshold for $P_{f}$ calculation, then always $P_{d}>P_{f}$.

Now we can study energy effectiveness case.

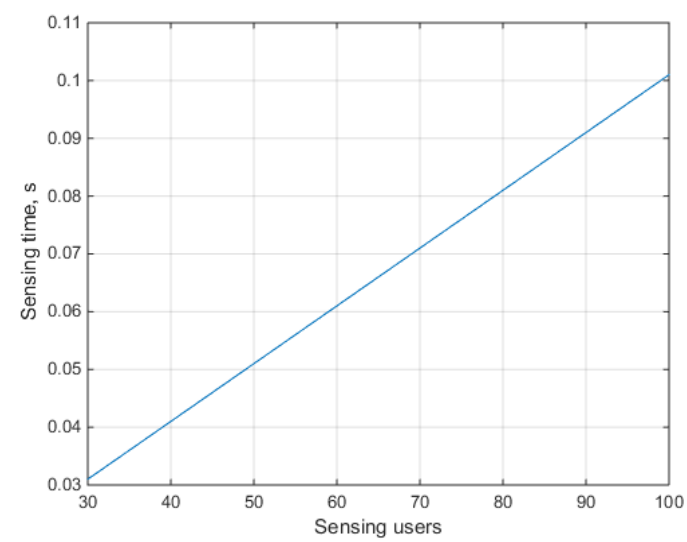

Figure 12. Sensing time for different SU number 


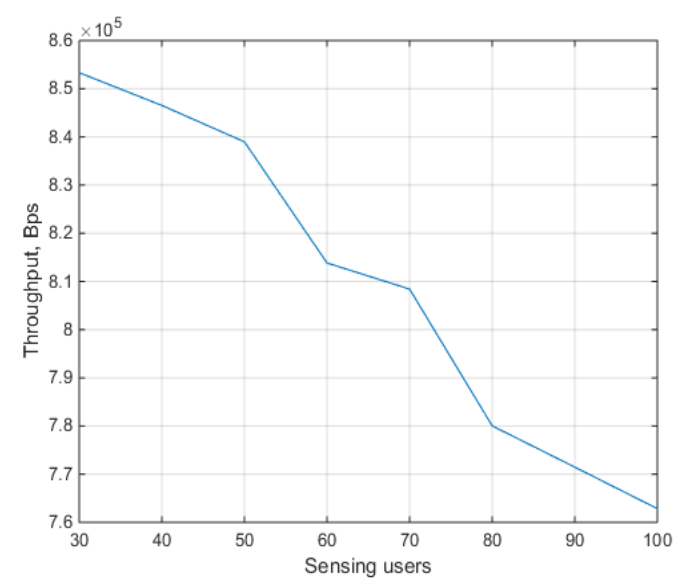

Figure 13. Total throughput at for different SU number

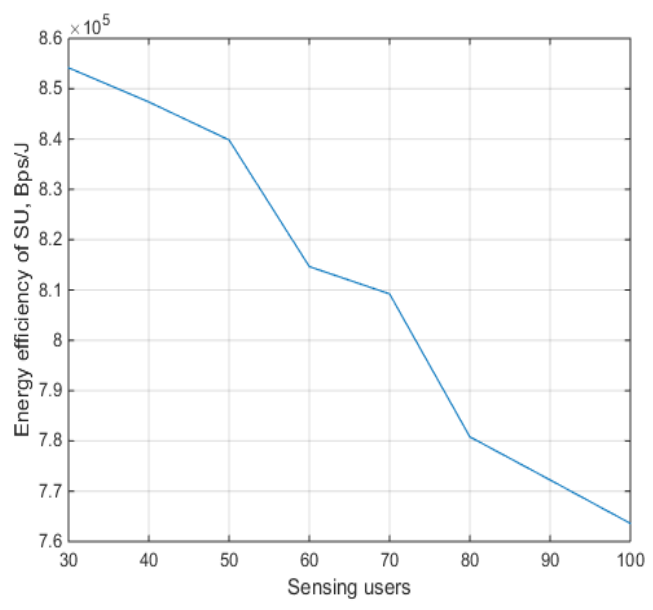

Figure 14. Energy efficiency of each SU for different SU number

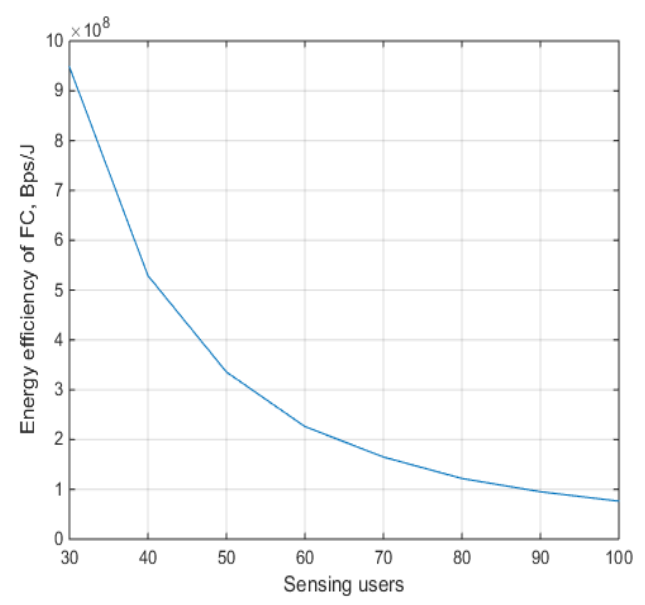

Figure 15. Energy efficiency of FC for different SU number

First of all we need to define time distribution according to (6). Cumulative sensing and report time for some number of $\mathrm{SU}$ if $T_{s}=T_{r}=1 \mathrm{~ms}$ while sensing is simultaneous and reporting to $\mathrm{FC}$ is provided with TDMA is shown in fig. 12. Another time is dedicated to data transmission between SU. Total throughput for SU can be calculated from eq. (7) where $P_{f}$ is taken from results of fig. $10(\mathrm{~b})$ and $P\left(H_{0}\right)=0.99, R=1 \mathrm{MBps}$. Results is shown in fig. 13 .
At least we can calculate energy effectiveness of SU and FC from (8) and (10) respectively with assumption that $w_{s}=$

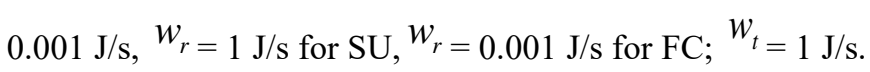
Results of these calculations are shown in fig 16 and fig. 17 respectively.

As we can see from fig. 14 and fig. 15 increasing of SU number decrease effectiveness of cooperative sensing while TDMA is using. Also we can predict that at other multiple access schemes this trend will be saved since $P_{f}$ performance is decreasing (value is increasing) while number of $\mathrm{SU}$ is increasing.

\section{CONCLUSION}

In this modern era, the $\mathrm{CR}$ is essential need for the transmission of data. Basically the licensed spectrum is only allocated for the licensed user for the transmission of data. To achieve higher data rates for transmission, cognitive radio technology is used. Cognitive radio technology is intelligent technology, which makes decision automatically. There are a lot of challenges faced by the $\mathrm{CR}$ in which one is spectrum sensing.

In this thesis, we focused on reducing the spectrum sensing time and increased the transmission time. Here we implement the K-Out-of-M rule, which is hard decision method to implement the algorithm in cognitive environment. The incremental voting mechanism is used for achieving the results. The proposed algorithm gives good results and work much efficient as compare to another spectrum decisions algorithm.

In this thesis, we focused on several mechanism for the spectrum sensing of $\mathrm{CR}$, while there are many portions on which the focus of work is needed on spectrum sensing, spectrum sharing etc. We can extend that work by adapting the voting mechanism in different environment and to overcome the probability of false alarm, we introduced the method which maintains the history on FC for those nodes who gave false alarm on every test.

\section{REFERENCES}

[1] Akyildiz IF, Lee WY, Vuran MC, Mohanty S. (2006). Next genera $\neg$ tion/dynamic spectrum access/cognitive radio wireless networks: a survey. Computer Networks 50(13): 2127-2159. https://doi.org/10.1016/j.comnet.2006.05.001

[2] Commission FC. (2003). Facilitating opportunities for flexible, efficient, and reliable spectrum use employing cognitive radio technologies, notice of proposed rule making and order, ET Docket 03-108.

[3] Mitola J. (2000). Cognitive radio - an integrated agent architecture for software defined radio.

[4] Commission FC. (2002). Spectrum policy task force report, fcc 02-155.

[5] Haykin S. (2005). Cognitive radio: brain-empowered wireless communications. Selected Areas in Communications, IEEE Journal 23(2): 201-220. http://doi.org/ 10.1109/JSAC.2004.839380

[6] Radhi NM. (2011). Implementation of spectrum sensing techniques for cognitive radio systems.

[7] Zhang W, Mallik RK, Letaief K. (2008). Cooperative spectrum sensing optimization in cognitive radio 
networks. in Communications, 2008. ICC'08. IEEE International Conference on. IEEE 3411-3415. http://doi.org/10.1109/ICC.2008.641

[8] Chen R, Park JM, Bian K. (2008). Robust distributed spectrum sensing in cognitive radio networks. in INFOCOM 2008. The 27th Conference on Computer Communications.

IEEE. http://doi.org/10.1109/INFOCOM.2008.251

[9] Chen R, Park JM, Hou YT, Reed JH. (2008). Toward secure distributed spectrum sensing in cognitive radio networks. Communications Magazine, IEEE 46(4): 50 55. http://doi.org/10.1109/MCOM.2008.4481340

[10] Letaief K, Zhang W. (2009). Cooperative communications for cognitive radio networks. Proceedings of the IEEE 97(5): 878-893. http://doi.org/10.1109/JPROC.2009.2015716

[11] Digham F, Alouini M, Simon M. (2003). On the energy detection of unknown signals over fading channels. IEEE International Conference on Communications 5: 35753579. http://doi.org/10.1109/ICC.2003.1204119

[12] Axell E, Leus G, Larsson EG, Poor HV. (2012). Spectrum sensing for cognitive radio. IEEE Signal Processing http://doi.org/10.1109/MSP.2012.2183771

[13] Olabiyi O, Alam S, Odejide O, Annamalai A. (2011). On the energy detection of unknown deterministic signals over generalized fading channel. Proc. of IWCN Conf. 2011, Las Vegas, USA. http://doi.org/10.1.1.217.9928

[14] Akyildiz IF, Lee WY, Vuran MC, Mohanty S. (2008). A survey on spectrum management in cognitive radio networks. IEEE Communications Magazine 46(4): 40-48. http://doi.org/10.1.1.217.9928

[15] Khalid L. (2014). Efficient techniques for cooperative spectrum sensing in cognitive radio networks. $\mathrm{PhD}$ thesis, School of Graduate Studies at Ryerson University, Toronto, Ontario, Canada, p. 206.

[16] Liang Y, Zeng Y, Peh EC, Hoang AT. (2008). Sensingthroughput trade of for cognitive radio networks. IEEE Transactions on Wireless Communications 7(4): 13261337. http://doi.org/10.1109/TWC.2008.060869

[17] Cardosa LS, Debbah M, Lasaulce S, Mari K, Palicot J. (2010). Spectrum sensing in cognitive radio networks. cogntive radio networks: architectures, protocols and standards. CRC Press, pp. 3-28.

[18] Goldsmith A, Jafar SA, Maric I, Srinivasa S. (2009). Breaking spectrum gridlock with cognitive radios: an information theoretic perspective. Proceedings of the IEEE 97(5): 894-914. http://doi.org/10.1109/TWC.2008.060869

[19] Kanti J, Tomar GS. (2016). Various sensing techniques in cognitive radio networks: A review. International Journal of Grid and Distributed Computing 9: 145-154. http://doi.org/10.14257/ijgdc.2016.9.1.15

[20] Awin FA, Abdel-Raheem E, Ahmadi M. (2014). Agile hierarchical cluster structure-based cooperative Spectrum sensing in cognitive radio networks. IEEE 26th International Conference in Microelectronics, ICM, Doha, Qatar pp. 48-51. http://doi.org/10.1109/ICM.2014.7071803 